\title{
Photoionization modeling of the Galactic planetary nebulae Abell 39 and NGC 7027
}

\author{
Ashkbiz Danehkar ${ }^{1}$, David J. Frew ${ }^{1}$, Quentin A. Parker ${ }^{1,2}$ and \\ Orsola De Marco ${ }^{1}$ \\ ${ }^{1}$ Department of Physics and Astronomy, Macquarie University, Sydney, NSW 2109, Australia \\ ${ }^{2}$ Australian Astronomical Observatory, PO Box 296, Epping, NSW 1710, Australia \\ email: ashkbiz.danehkar@mq.edu.au; david.frew@mq.edu.au
}

\begin{abstract}
We estimate distances to the spherical planetary nebula Abell 39 and the bipolar planetary nebula NGC 7027 by interpolating from a wide grid of photoionization models using the 3-D code, MOCASSIN. We find preliminary distances of $1.5 \mathrm{kpc}$ and $0.9 \mathrm{kpc}$ respectively, with uncertainties of about $30 \%$.
\end{abstract}

Keywords. planetary nebulae: individual (Abell 39, NGC 7027)

\section{Introduction}

Accurate distances to planetary nebulae $(\mathrm{PNe})$ are crucial in unraveling the connection between the physical properties of the nebulae with those of their central stars (CSs). Reliable distances facilitate the accurate estimation of fundamental parameters such as the CS mass and luminosity, and the nebular mass and age. We have begun a program of using photoionization modeling to refine the distances to a sample of nearby $\mathrm{PNe}$ (see also Danehkar et al. 2011). In this work, we study two very different $\mathrm{PNe}$ as a proof of concept: Abell 39, a simple spherical shell with no microstructures, and NGC 7027, a well-known, young, luminous bipolar PN with a massive molecular envelope. Using the 3-D photoionization code (MOCASSIN; Ercolano et al. 2003), our ultimate aim is to constrain the distance to individual $\mathrm{PNe}$, utilizing the physical $\mathrm{PN}$ radius we have calculated and the angular size.

\section{Analysis}

Our procedure allows us refine the nebular parameters iteratively to provide the best match against the observations, though owing to degeneracies in these parameters, there may be more than one unique solution. Since a black body is not a perfect model for the CS continuum flux, we used various NLTE model atmosphere fluxes from the grid of Rauch (2003). For the initial model inputs for A 39, we adopt the line intensities and abundances from Jacoby et al. (2001), and derived the nebular $T_{\mathrm{e}}$ from the [OIII] lines Because the density of A 39 is very low, we initially determined $N_{e}$ from the PN diameter and integrated $\mathrm{H} \beta$ flux. Our preliminary distance estimate is $D=1.5 \mathrm{kpc}$, and the CS parameters are $T_{\text {eff }}=160 \mathrm{kK}$ and $L / L_{\odot}=1800$, which disagrees with the estimate of $T_{\text {eff }}=117 \mathrm{kK}$ from Ziegler (2011, these proceedings). The higher temperature is needed to explain the observed He II and [Ne v] line intensities in the nebular shell.

For NGC 7027, a starting value of $T_{\mathrm{e}}=11 \mathrm{kK}$ is adopted, the line intensities and abundances being taken from Bernard Salas et al. (2001) and Zhang et al. (2005), and refined as necessary. In addition, a bipolar morphology with a mean density $\left(n_{\mathrm{H}}=\right.$ $55,000 \mathrm{~cm}^{-3}$; Fig. 1) is needed to model NGC 7027, since a simple assumption of spherical 
Table 1. Best-fit parameters (left) and observations versus model outputs (right).

\begin{tabular}{|c|c|c|}
\hline Parameter & A 39 & NGC 7027 \\
\hline$T_{\text {eff }}(\mathrm{K})$ & 160000 & 180000 \\
\hline$L_{\mathrm{cs}}\left(\mathrm{L}_{\odot}\right)$ & 1800 & 7500 \\
\hline$M_{*}\left(\mathrm{M}_{\odot}\right)$ & 0.59 & 0.69 \\
\hline$R_{\text {out }}(\mathrm{pc})$ & 0.64 & 0.022 \\
\hline$\delta r_{\text {shell }}(\mathrm{pc})$ & 0.07 & 0.015 \\
\hline$D(\mathrm{pc})$ & 1500 & 900 \\
\hline$n_{\mathrm{H}}\left(\mathrm{cm}^{-3}\right)$ & 36 & 55000 \\
\hline$T_{e}(\mathrm{~K})$ & 15400 & 16400 \\
\hline$\varepsilon$ & 0.45 & 0.50 \\
\hline $\log (\mathrm{He} / \mathrm{H})$ & -1.02 & -1.0 \\
\hline $\log (\mathrm{N} / \mathrm{H})$ & -4.30 & -3.89 \\
\hline $\log (\mathrm{O} / \mathrm{H})$ & -3.76 & -3.52 \\
\hline $\log (\mathrm{Ne} / \mathrm{H})$ & -4.45 & -4.15 \\
\hline $\log (\mathrm{S} / \mathrm{H})$ & -5.19 & -5.08 \\
\hline $\log (\mathrm{Ar} / \mathrm{H})$ & -6.05 & -5.80 \\
\hline
\end{tabular}

\begin{tabular}{|c|c|c|c|c|c|}
\hline \multicolumn{2}{|l|}{ Line } & \multicolumn{2}{|c|}{ A 39} & \multicolumn{2}{|c|}{ NGC 7027} \\
\hline Ion & $\lambda(\AA)$ & Obs. & Mod. & Obs. & Mod. \\
\hline$[\mathrm{Ne} \mathrm{V}]$ & 3426 & 9.8 & 8.0 & 154 & 139 \\
\hline$\left[\begin{array}{ll}\mathrm{O} & \mathrm{II}\end{array}\right]$ & 3727 & 40 & 51 & 21 & 21 \\
\hline [Ne III] & 3869 & 104 & 92 & 126 & 143 \\
\hline $\mathrm{H} \gamma$ & 4340 & 48 & 47 & 47 & 48 \\
\hline$\left[\begin{array}{ll}\mathrm{O} & \mathrm{III}\end{array}\right]$ & 4363 & 24 & 20 & 25 & 39 \\
\hline He II & 4686 & 95 & 94 & 49 & 62 \\
\hline$\left[\begin{array}{ll}\text { Ar } & \text { IV }\end{array}\right]$ & 4740 & 4.0 & 4.5 & 8.1 & 8.1 \\
\hline $\mathrm{H} \beta$ & 4861 & 100 & 100 & 100 & 100 \\
\hline$\left[\begin{array}{ll}\mathrm{O} & \mathrm{III}\end{array}\right]$ & 5007 & 1131 & 957 & 1397 & 1345 \\
\hline$[\mathrm{N}$ II] & 5755 & & 1.4 & 5.8 & 7.2 \\
\hline He I & 5876 & 1.9 & 1.9 & 10.9 & 7.5 \\
\hline $\mathrm{H} \alpha$ & 6563 & 286 & 278 & 285 & 275 \\
\hline$\left[\begin{array}{ll}\mathrm{N} & \mathrm{II}\end{array}\right]$ & 6584 & 12 & 41 & 110 & 118 \\
\hline$[\mathrm{S}$ II $]$ & 6724 & 9.7 & 11 & 5.6 & 5.3 \\
\hline$\left[\begin{array}{ll}\text { Arr } & \text { III }\end{array}\right]$ & 7135 & 5.8 & 2.2 & 21 & 8 \\
\hline$L_{\mathrm{H} \beta}(\mathrm{erg} / \mathrm{s})$ & 1E33 & 0.46 & 0.50 & 131 & 128 \\
\hline
\end{tabular}
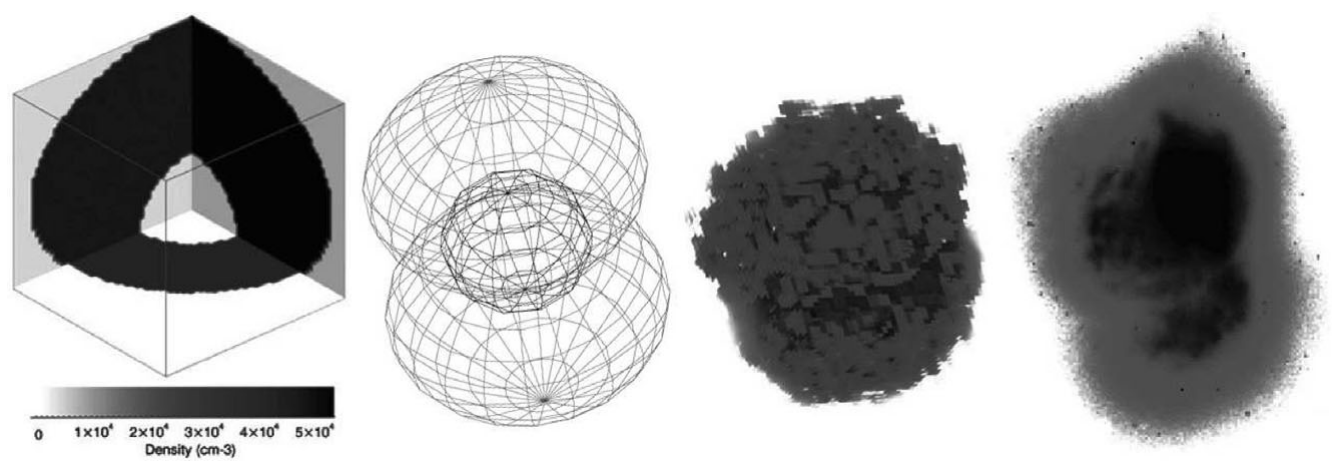

Figure 1. (Left) Density distribution, cross section, and morphology used for NGC 7027. (Right) Computed surface brightness in the He II $\lambda 4686$ line compared with the HST image.

geometry conflicts with the observed ionization structure and nebular line intensities. Our photoionization model outputs generally agree with the observations, except for some uncertain lines. Our photoionization model of NGC 7027 gives $D=900 \mathrm{pc}$, with the CSPN having $T_{\text {eff }}=180 \mathrm{kK}$ and $L / L_{\odot}=7500$, which reproduces most observations. Our distance agrees with previous determinations (Masson 1989; Volk \& Kwok 1997; Zijlstra et al. 2008).

\section{Acknowledgements}

AD acknowledges receipt of an MQRES PhD Scholarship and an IAU Travel Grant.

\section{References}

Bernard Salas, J., Pottasch, S. R., Beintema, D. A., \& Wesselius, P. R. 2001, A\& A, 367, 949 Danehkar, A., Frew, D. J., Parker, Q., \& De Marco, O. 2011, IAUS 281, in press, arXiv:1109.2181 Ercolano, B., Barlow, M. J., Storey, P. J., \& Liu, X. W. 2003, MNRAS, 340, 1153

Jacoby, G. H., Ferland, G. J., \& Korista, K. T. 2001, ApJ, 560, 272

Masson, C. R. 1989, ApJ, 336, 294

Rauch, T. 2003, A\&\&A, 403, 709

Volk, K. \& Kwok, S. 1997, ApJ, 477, 722

Zhang, Y., Liu, X. W., Luo, S. G., Péquignot, D., \& Barlow, M. J. 2005, A $\& A, 442,249$

Zijlstra, A. A., van Hoof, P. A. M., \& Perley, R. A. 2008, ApJ, 681, 1296 